\title{
'ANÁLISIS DE TRANSFERENCIAS LINGÜÍSTICAS POSITIVAS Y NEGATIVAS EN EL PLANO LÉXICO-SEMÁNTICO ENTRE EL INGLÉS (L2) Y EL ALEMÁN (L3) PARA TURISMO
}

\author{
Alfonso Corbacho Sánchez \\ Universidad de Extremadura
}

Die Gewalt einer Sprache ist nicht, dass sie das Fremde abweist, sondern dass sie es verschlingt.

Goethe

\begin{abstract}
The main objective of this article is to examine a question that is becoming more and more important in modern language teaching: linguistic transfers from English as a second language to German as a third language. This is particularly pertinent to the discussion of learning these languages for tourism. With this aim in mind, the first part of the study starts by briefly describing a theoretical framework for understanding the nature and function that transfers play in the L3 learning process. This is followed by a discussion of examples provided by students enrolled in a Degree on Tourism. These real data are examined from a lexical point of view in order to prove the importance that linguistic phenomena such as positive and negative transfer have in learning German as a third language. Finally, some consideration is given to the attitudes of learners in this context in which it is obvious that students take advantage of the cases described here and that will help them improve their learning.
\end{abstract}

\section{A MODO DE INTRODUCCIÓN}

No es ninguna novedad sostener que una elevada proporción de alumnos al simultanear el estudio de las lenguas inglesa y alemana sufren en esta última frecuentes interferencias con el inglés; y máxime cuando nos remitimos a un alumnado principiante que ya cuenta con un bagaje considerable de conocimientos de la lengua inglesa por ser ésta precisamente la primera opción y la más frecuente en el currículo de lenguas extranjeras de la educación primaria y secundaria. Si nos ceñimos al ámbito universitario, un colectivo estudiantil 
representativo de las circunstancias expuestas lo configura el alumno hispanohablante matriculado en la diplomatura de Turismo.

Sin embargo, no todas las interacciones del inglés serán de naturaleza negativa. Estamos convencidos, como quedará contrastado más adelante, de que la lengua anglosajona puede resultar un apoyo más en el aprendizaje del alemán.

Por lo tanto, el ámbito de investigación del presente trabajo está delimitado no sólo al estudio de los errores interferenciales cometidos en el alemán (L3) por la influencia del inglés (L2) en estudiantes que comparten la misma lengua materna -el castellano-, sino también a las interferencias de efecto positivo concentrándonos en esta situación de simultaneidad de dos lenguas extranjeras única y exclusivamente en el plano léxicosemántico.

En lo que concierne a las muestras que someteremos a examen, proceden en su inmensa mayoría de producciones orales y escritas recopiladas a lo largo de nuestra trayectoria docente por distintas Escuelas Universitarias en las que hemos impartido las asignaturas de "Alemán Turístico I, II, III" de la correspondiente diplomatura de Turismo. En este sentido, antes de proseguir con nuestra contribución, es conveniente insistir en que para la exposición de las muestras aportadas no contamos con un estudio de frecuencias ni con una amplia recopilación de datos, basándonos principalmente en nuestra experiencia como docentes y en la intuición como lingüistas.

\section{FUNDAMENTOS TEÓRICOS: DESCRIPCIÓN DEL CONCEPTO DE TRANSFERENCIA LINGÜÍSTICA}

Dado que el presente trabajo gira en torno a las transferencias lingüísticas, es primordial, en primer lugar, acotar los límites de dicha noción desde un punto de vista estrictamente teórico.

La mayor carga porcentual de los estudios realizados en lo relativo a la influencia de una lengua sobre el aprendizaje ${ }^{1}$ de otra nueva han tomado como punto de referencia los fenómenos lingüísticos que se transfieren de la lengua materna o L1 ampliado a una L2. Pues bien, estas huellas de la lengua materna se conciben como transferencias, que, a su vez, pueden dividirse en:

a) Transferencia positiva, entendiendo como tal la que "facilita el aprendizaje, y puede darse cuando la lengua nativa y la lengua meta tienen la misma forma" (Richards et al., 1997: 420)

b) Transferencia negativa que también se identifica con el concepto de interferencia y se define como "el uso de una construcción o regla de la lengua nativa que conduce

\footnotetext{
${ }^{1}$ Coincidimos en este contexto plenamente con la opinión de Hufeisen (2000) al diferenciar las nociones de "adquisición" y "aprendizaje". Pues bien, con el término de adquisición describe el aprendizaje natural de la lengua materna, mientras que con aprendizaje se refiere al estudio del sistema lingüístico de una L2 o L3 en todos sus aspectos, es decir, gramatical, léxico, fonético, etc.
} 
a un ERROR o forma inapropiada de la LENGUA META" (Richards et al., 1997: 419).

Sin embargo, no todos los casos tipificados como transferencias negativas o interferencias obedecen a una misma motivación. Así lo afirman con rotundidad Alcaraz y Martínez (1997: 303) en la descripción que nos proporcionan sobre el concepto de interferencia:

'La 'interferencia' consiste fundamentalmente en la utilización en una lengua de elementos léxicos, morfológicos, sintácticos y/o pragmáticos específicos de otra lengua distinta".

Menos explícito y más genérico en la descripción del término es el estudioso Juhász (1970: 9), uno de los primeros cultivadores de la materia que está siendo objeto de análisis. Pues bien, entiende por interferencia la lesión de un sistema lingüístico o norma lingüística provocada por la influencia de otros elementos y reglas lingüísticos. Dicho con sus palabras:

"[...] die durch die Beeinflussung von anderen sprachlichen Elementen und Regeln - verursachte Verletzung eines Sprachsystems oder einer sprachlichen Norm [...]"

Asimismo, se desprende de las investigaciones llevadas a cabo sobre el tema aquí tratado que la manera de aprender una lengua extranjera como L3 es diferente al modo en que lo hacemos como L2. En esta dirección, cabe apuntar que los alumnos bilingües gozan de mayores ventajas y están más motivados a la hora de aprender una L3 que aquellos otros aprendices que se enfrentan por primera vez al estudio de una lengua extranjera (vid. Thomas, 1988; Köberle, 1998). Por tanto, podemos establecer que el dominio de varios idiomas proporciona ventajas adicionales y facilita el aprendizaje de nuevas lenguas extranjeras por un mecanismo que asocia la materia novedosa con los sistemas lingüísticos ya aprendidos ${ }^{2}$.

En definitiva, tal como se ha indicado más arriba, abordaremos en las páginas que siguen el análisis de las transferencias lingüísticas desde la perspectiva léxico-semántica, pero no de la lengua materna sobre otra lengua extranjera, sino de una L2, el inglés, a una L3, el alemán ${ }^{3}$. No es necesario recalcar que no entraremos en el análisis de las transferencias que puedan originarse en el sentido inverso, esto es, del alemán como L2 en el inglés como L3.

\footnotetext{
${ }^{2}$ Destacable es el trabajo de Meisel (1983) en un intento de explicar el fenómeno de las transferencias de una L2 en la L3. El autor hace referencia a la manera en que el cerebro procesa las lenguas extranjeras (L2-L3) en oposición al modo en que se procesa la lengua materna o L1.

${ }^{3}$ Para más información acerca de las transferencias del inglés L3 en el alemán L2 son fundamentales los trabajos de Welge (1987), Hufeisen (1991), Vogel (1992), Neuner (1996) y Dentler (2000). Otros estudios interesantes de carácter más general acerca de la influencia de una L2 en una L3 y sobre el aprendizaje de una tercera lengua son Vrient (1972), Stedje (1976), Bausch / Heid (1990), Hammarberg / Williams (1993), Sikogukira (1993), Klein (1995) y Hufeisen $(1999,2000,2001)$.
} 


\section{ESTUDIO EMPÍRICO DE LAS TRANSFERENCIAS LÉXICO-SEMÁNTICAS INGLÉS-ALEMÁN EN CONTEXTOS TURÍSTICOS}

Las transferencias entre una L2 y una L3, ya sean positivas o negativas, son más probables que se produzcan en lenguas con un alto grado de relación o similitud entre sí (vid. Vrient, 1972; Dentler, 1998) como es el caso de las lenguas de origen germánico: el inglés y el alemán. Efectivamente, el alemán pertenece al grupo de las lenguas indoeuropeas, que, al mismo tiempo, se incluye en el grupo occidental de las lenguas identificadas como germánicas y que, por consiguiente, está lingüísticamente emparentado con el danés, el sueco, el noruego, el holandés, el flamenco y, por lo que aquí nos interesa, el inglés.

De hecho, si nos atenemos a factores socioculturales (cfr. Singh / Carroll, 1979), el alumno de alemán (L3) puede encontrar una mayor proximidad y afinidad con el inglés (L2) que con el castellano (L1), circunstancia que repercute indiscutiblemente de forma positiva en su aprendizaje.

Así, los aciertos y errores de nuestros alumnos en alemán (L3) provienen, en su inmensa mayoría, de sus conocimientos del inglés (L2) y no del español, lengua que no presenta ninguna base histórica común con el idioma objeto de aprendizaje. Es un hecho contrastado que el alumno ante situaciones que le resultan problemáticas, tanto a efectos de comprensión como producción oral, tiende automáticamente a emplear el inglés antes de recurrir al castellano para solventar el citado problema. Y todo ello, no sólo dentro de los muros del aula sino también ante cualquier situación cotidiana en la que tenga que utilizar el alemán como lengua de comunicación.

Por otro lado, es el plano léxico-semántico el principal referente que nos permite divisar un mayor número de transferencias del inglés (L2) en el alemán (L3) por encima de otros niveles como el de la sintaxis (vid. Vogel, 1992), en el que el aprendiz distingue con más facilidad los sistemas lingüísticos de las dos lenguas.

Sin más dilaciones, procedemos seguidamente a ejemplificar casos representativos de lo que entendemos por transferencias lingüísticas en contextos propios del sector turístico, sin entrar para ello en aclaraciones pormenorizadas de carácter gramatical en cada uno de los términos expuestos. Por último, no es necesario hacer hincapié en que nuestra exposición no pretende ser descriptivamente exhaustiva, a pesar de que hemos logrado reunir una cantidad muy nutrida y representativa de ejemplos.

\subsection{Transferencias positivas}

Comenzaremos clasificando los ejemplos por su pertenencia a diferentes campos temáticos estrechamente relacionados con el sector turístico, al menos debemos tomar en consideración que figuran como puntos cardinales en cualquier manual didáctico de alemán como lengua extranjera para fines turísticos. En todas las muestras el primer término corresponde al inglés seguido del vocablo equivalente en lengua alemana.

- Hotel-Alojamiento 
address - Adresse, alarm - Alarm, balcony - Balkon, bath - Bad, bed - Bett, bungalow - Bungalow, garage - Garage, hotel - Hotel, house - Haus, information Information, lamp - Lampe, luxury hotel - Luxushotel, price - Preis, radio - Radio, safe - Safe, sofa - Sofa, telephone - Telefon, toilet - Toilette, tourist - Tourist.

- Viajes

boat - Boot, bus - Bus, charter flight - Charterflug, ferry - Fähre, helicopter Helikopter, jet - Jet, kilometre - Kilometer, moped - Moped, pilot - Pilot, ship Schiff, station - Station, taxi - Taxi, tunnel - Tunnel.

- $\quad$ Arte y Cultura

altar - Altar, antique - Antiquität, catedral - Kathedrale, concert - Konzert, diamond - Diamant, museum - Museum, palace - Palast, theatre - Theater, vase Vase.

- PAÍSES y CONTINENTES

America - Amerika, Australia - Australien, Canada - Kanada, China - China, continent - Kontinent, Denmark - Dänemark, England - England, Europe - Europa, Holland - Holland, Iceland - Island, Italy - Italien, Japan - Japan, Luxembourg Luxemburg, Portugal - Portugal, Scotland - Schottland, Sweden - Schweden, Thailand - Thailand, Ukraine - Ukraine.

- GASTRONOMÍA

apple - Apfel, banana - Banane, bread - Brot, butter - Butter, carrots - Karrots, chocolate - Schokolade, cocktail - Cocktail, fish - Fisch, grill - Grill, hamburger Hamburger, hunger - Hunger, hungry - hungrig, ice - Eis, kiwi - Kiwi, mango mango, margarine - Margarine, melon - Melone, mussel - Muschel, orange Orange, restaurant - Restaurant, salad - Salat, salt- Salz, sandwich - Sandwich, soup - Suppe, spinach - Spinat, steak - Steak, toast - Toast, tomato - Tomate, walnut - Walnuss, yoghurt - Joghurt.

- BEBIDAS

beer - Bier, camomile tea - Kamillentee, coffee - Kaffee, glass - Glas, grapefruit Grapefruit, lemonade - Limonade, milk - Milch, sherry - Sherry, tea - Tee, water Wasser, wine - Wein..

- Parque Zoológico

Aquarium - Aquarium, bear - Bär, camel - Kamel, cat - Katze, cow - Kuh, crocodile - Krokodil, dolphin - Delphin, elephant - Elephant, frog - Frosch, giraffe Giraffe, gorilla - Gorilla, hamster - Hamster, jaguar - Jaguar, kangaroo - Kanguru, mouse - Maus, rat - Ratte, swan - Schwan, tiger - Tiger, zebra - Zebra, zoo - Zoo.

- DEPORTES

badminton - Badminton, basketball - Basketball, football - Fußball, golf - Golf, ice hockey - Eishockey, jogging - Jogging, judo - Judo, karate - Karate, ski - Ski, tennis - Tennis, windsurfing - Windsurfen.

- OCIO 
artist - Artist, billiards - Billard, cards - Karten, casino - Kasino, clown - Clown, club - Club, dance - Tanz, domino - Domino, film - Film, hobby - Hobby, jazz Jazz, music - Musik, party - Party.

- NATURAlezA

garden - Garten, grass - Gras, land - Land, nature - Natur, park - Park, rose Rose, sand - Sand.

- EXPRESIONES DE TIEMPO

minute - Minute, monday - Montag, friday - Freitag, January - Januar, February Februar, april - April, may - Mai, june - Juni, july - Juli, august - August, september September, october - Oktober, november - November, december Dezember, summer - Sommer, winter - Winter.

- Otros

arm - Arm, bank - Bank, bikini - Bikini, bring - bringen, brother - Bruder, brown - Braun, calendar - Kalender, camera - Kamera, can - kann (können), catalogue Katalog, cigarette - Zigarette, computer - Computer, father - Vater, fever - Fieber, finger - Finger, gold - Gold, good - gut, hand - Hand, here - hier, hundred Hundert, hunger - Hunger, jeans - Jeans, job - Job, knee - Knie, long - lang, man - Mann, massage - Massage, mild - Mild, mother - Mutter, old - alt, packet Paket, paper - Papier, person - Person, press - Presse, ring - Ring, shampoo Shampoo, shoe - Schuh, silver - Silber, son - Sohn, supermarket - Supermarkt, thousand - Tausend, walkman - Walkman, warm - warm, weather - Wetter, wild wild, young - jung,

Como ha podido comprobarse, las muestras expuestas facilitan el aprendizaje del léxico del alemán como tercera lengua y no exclusivamente por su similitud ortográfica, casi total en gran parte de los ejemplos, sino también, en algunos casos, por su analogía fonológica si bien ambas circunstancias pueden conducirnos a error en determinadas situaciones ${ }^{4}$-, pero, sobre todo, por su identificación semántica. Así pues, no nos cabe la menor duda de que estamos ante pruebas fehacientes, cuando menos en el plano léxico-semántico, de lo que entendemos por transferencias positivas.

\subsection{Transferencias negativas}

Sobresalen en este apartado básicamente los errores que se originan por la similitud formal que existe entre los términos ingleses y alemanes. Por consiguiente, nos vemos obligados en este apartado de las transferencias negativas a dilucidar el concepto de falsos amigos (vid. Dentler, 1998; Hufeisen, 1991: 92 y sigs.). Éstos hacen referencia a los

\footnotetext{
${ }^{4}$ Es importante recalcar una vez más que, por una parte, los ejemplos pueden contribuir a potenciar los conocimientos léxicos, pero, por otra parte, también pueden provocar interferencias -transferencias negativas- al obstaculizar el aprendizaje de la ortografía y la pronunciación. Valga como aclaración el vocablo del inglés bed (cama) frente a Bett (cama) en alemán. La analogía ortográfica puede provocar el consiguiente error, aunque en el plano semántico ambos conceptos se refieren a la misma realidad, facilitando desde esta postura el aprendizaje de la L3.
} 
vocablos de dos lenguas distintas que coinciden en su apariencia externa o son muy similares, pero con obvias discrepancias en el terreno de la semántica dado que corresponden a dos realidades diferentes. El estudioso Gerd Wotjak (1996: 125 y sig.) perfila con claridad y precisión que los falsos amigos son:

“... unidades léxicas (UL) que se caracterizan por coincidencias totales o parciales en lo formal, o sea, en el plano de la expresión y en lo semántico comunicativo, o sea, en el plano del contenido, y que se destacan al mismo tiempo por divergencias más o menos considerables en uno de estos dos planos constitutivos para cualquier UL como signo lingüístico bilateral."

Hecha esta puntualización, damos paso a los casos más frecuentes, sin pretensión de exhaustividad, de las transferencias negativas que el aprendiz de alemán tiende a utilizar. Al igual que en el apartado anterior, el primer vocablo corresponde al inglés acompañado de su interpretación errónea en alemán y entre paréntesis adjuntamos la traducción de ambos términos al español:

- also (también) / also (por lo tanto, así)

- bald (calvo) / bald (pronto)

- become (llegar a ser, convertirse en) / bekommen (obtener, recibir)

- $\quad$ brief (breve) / Brief (carta, correspondencia)

- dome (cúpula)/Dom (catedral)

- dish (plato) / Tisch (mesa)

- eagle (águila) / Igel (erizo)

- fast (rápido) / fast (casi)

- flash (relámpago) / Flasche (botella)

- floor (suelo) / Flur (pasillo)

- gift (regalo) / Gift (veneno)

- ground (suelo) / Grund (motivo)

- guilty (culpable) / gültig (válido)

- hell (infierno) / hell (claro)

- hut (cabaña)/ Hut (sombrero)

- $\quad$ kind (especie, clase) / Kind (niño/a)

- lager (cerveza) / Lager (almacén)

- loan (préstamo) / Lohn (salario)

- $\operatorname{map}$ (mapa) / Mappe (carpeta)

- man (hombre) / man (pronombre indefinido cuya traducción puede ser "uno/a" o "se")

- $\quad$ тепu (la carta del restaurante) / Menü (menú)

- $\quad$ mist (niebla) / Mist (estiércol, basura)

- photograph (fotografía) / Fotograf (fotógrafo)

- roman (romano) / Roman (novela)

- $\operatorname{see}$ (ver) / See (mar, lago) 
- sister (hermana) / Geschwister (hermanos, plural válido tanto para el masculino como para el femenino)

- $\operatorname{slim}$ (delgado) / schlimm (mal)

- small (pequeño) / schmal (estrecho)

- $\quad$ stern (severo) / Stern (estrella)

- $\quad$ still (aún, todavía) / still (tranquilo)

- send (enviar) / senden (también "enviar", pero puede significar "emitir por televisión")

- $\quad$ stock (existencias) / Stock (piso o planta de un edificio)

- $\quad$ stool (taburete) / Stuhl (silla)

- then (entonces) / denn (pues)

- wander (vagar) / wandern (ir de excursión)

- When? (¿cuándo?) / wenn (cuando -conjunción temporal-, si -conjunción condicional-)

- Where? (¿dónde?) / Wer? (¿quién?)

- Who? (¿quién?)/ Wo? (¿dónde?)

- wide (ancho) / weit (lejos)

- will (auxiliar de futuro) / will ( $1^{\mathrm{a}}$ y $3^{\mathrm{a}}$ persona del singular del verbo modal wollen $=$ querer $)$

\section{CONSIDERACIONES FINALES}

Tras el análisis contrastivo emprendido, no podemos negar la existencia de una amplia zona reservada a las transferencias bajo el poderoso influjo anglosajón en temas relacionados con el mundo del turismo. En la mayor parte de los casos expuestos podemos observar que el empleo de los términos no experimenta ninguna modificación en lo que atañe a su estructura formal.

Por otro lado, los datos aportados suponen un enriquecimiento a la labor docente y al alumnado, especialmente en lo referente a la previsión de errores interferenciales para que así puedan ser superados con mayor facilidad. En efecto, los alumnos deben ser conscientes de la existencia de estas interferencias al objeto de evitar que determinadas palabras sean utilizadas en ambas lenguas para un mismo contexto. Asimismo, la distinción y localización de este fenómeno contribuye a un conocimiento más profundo de los dos idiomas de manera que el estudiante pueda aplicar con corrección el léxico de L3, que en el presente estudio constituye el objetivo prioritario.

En definitiva, según lo expuesto, podemos establecer que los conocimientos previos del inglés en el aprendizaje del alemán son en todo momento rentables, pues permiten detectar con mayor facilidad las interferencias y, a su vez, posibilitan el aprovechamiento de las transferencias positivas. No olvidemos tampoco que no debe ser el propósito de estos estudios $\mathrm{y}$, por ende, del contexto educativo la anulación total y absoluta de los conocimientos de la lengua inglesa para sustituirlos por los nuevos contenidos de la lengua alemana. Ambos idiomas deben ser aprovechados y más en los albores del siglo XXI en la que la figura del políglota se ha convertido en un status profesional muy cotizado. 
Un detalle más que no podemos dejar escapar se basa en el tipo de las transferencias, al margen de que nos hayamos ceñido al estudio léxico-semántico, creemos firmemente que la interacción positiva y negativa en los diferentes ámbitos (fonético, léxico-semántico, morfológico y sintáctico), tal y como hemos indicado más arriba, puede y debe entenderse mejor desde una perspectiva integradora y no excluyente. Esperamos, por otro lado, que todo lo expuesto en este sucinto análisis, a pesar de no ser novedoso, sí resulte útil para despejar las posibles dudas que el concepto de transferencia (interferencia) pudiera ofrecer $\mathrm{y}$, sobre todo, para sensibilizarnos de la gran utilidad que puede tener, entre otras parcelas de la lingüística, en la didáctica de las lenguas extranjeras, ya sean éstas de carácter general o especializado.

Para concluir, no ha sido la única pretensión de este trabajo manifestar nuestra inquietud sobre el amplio tema de las transferencias, sino también poder abrir otras líneas de investigación en torno a las transferencias que profundicen en otros campos profesionales y de especialización, dada la relevancia de todos los fenómenos lingüísticos que acontecen en la sociedad multilingüe de nuestros días en la que las lenguas están cada vez más interconectadas.

\section{REFERENCIAS BIBLIOGRÁFICAS}

ALCARAZ VARÓ, E./MARTÍNEZ LINARES, M. A., Diccionario de lingüistica moderna, Barcelona, Ariel, 1997.

BAUSCH, K.-R./HEID, M., (eds.), Das Lehren und Lernen von Deutsch als zweiter oder weiterer Fremdsprache: Spezifika, Probleme, Perspektiven, Bochum, Universitätsverlag Brockmeyer, 1990.

BURGSCHMIDT, E./GÖTZ, D., Kontrastive Linguistik Deutsch/Englisch. Theorie und Anwendung, München, Hueber, 1974.

DENTLER, S., "Zur Systematizität und Prognostizierbarkeit lexikalischer Interferenzen". En: B. Hufeisen / B. Lindemann (eds.), Tertiärsprachen. Theorien, Modelle, Methoden, Tübingen, Stauffenburg, 1998, pp. 31-46.

DENTLER, S. "Deutsch und Englisch - das gibt immer Krieg!". En: S. Dentler / B. Hufeisen / B. Lindemann (eds.), Tertiär- und Drittsprachen: Projekte und empirische Untersuchungen, Tübingen, Stauffenburg, 2000, pp. 77-97.

HAMMARBERG, B./WILLIAMS, S., "A study of Third Language Acquisition”. En: B, Hammarberg (ed.), Problem, process, product in language learning, Stockholm, Stockholm University, 1993, pp. 60-69.

HUFEISEN, B., Englisch als erste und Deutsch als zweite Fremdsprache: empirische Untersuchung zur fremdsprachlichen Interaktion, Frankfurt, Lang, 1991.

HUFEISEN, B., "Deutsch als zweite Fremdsprache", Fremdsprache Deutsch 20 (1999), pp. 4-6.

HUFEISEN, B, “A European Perspective: Tertiary Languages with a Focus on German as L3". En: J.-W. Rosenthal, Handbook of Undergraduate Second Language Learning Education: English as a Second Language, Bilingual and Foreign 
Language Instruction for a Multilingual World, Mahwah-New Jersey, Erlbaum, 2000, pp. 209-229.

HUFEISEN, B., "Deutsch als Tertiärsprache". En: G. Helbig et al., Deutsch als Fremdsprache. Ein internationales Handbuch, Berlin: de Gruyter, 2001, pp. 648-653.

KLEIN, E., "Second vs. third language acquisition: Is there a difference?", Language Learning, 3 (1995), pp. 419-465.

KÖBERLE, B., "Positive Interaktion zwischen L2, L3 und L4 und ihre Applikabilität im Fremdsprachenunterricht". En: B. Hufeisen / B. Lindemann (eds.), Tertiärsprachen. Theorien, Modelle, Methoden, Tübingen, Stauffenburg, pp. 89109.

JUHÁSZ, J., Probleme der Interferenz, München, Hueber, 1970.

HELLINGER, M., Kontrastive Grammatik Deutsch/Englisch, Tübingen, Niemeyer, 1977.

MEISEL, J., "Transfer as a second language strategy", Language and Communication, 3 (1983), pp. 11-46.

NEUNER, G., "Deutsch als zweite Fremdsprache nach Englisch. Überlegungen zur Didaktik und Methodik und zur Lehrmaterialentwicklung für die 'Drittsprache Deutsch' ", Deutsch als Fremdsprache, 4 (1996), pp. 211-217.

RICHARDS, J. C./Platt, J./Platt, H., Diccionario de lingüística aplicada y enseñanza de lenguas, Barcelona, Ariel, 1997.

SIKOGUKIRA, M., "Influence of languages other than the L1 on a foreign language: a case of transfer from L2 to L3", Edinburgh working papers in applied linguistics, 4 (1993), pp. 110-132.

SINGH, R./CARROLL, S., "L1, L2, and L3", Indian Journal of Applied Linguistics, 5, 1 (1979), pp. 51-63.

STEDJE, A., "Interferenz von Muttersprache und Zweitsprache auf eine dritte Sprache beim freien Sprechen - ein Vergleich", Zielsprache Deutsch 1 (1976), pp. 15-21.

THOMAS, J., "The role played by metalinguistic awareness in second and third language learning”, Journal of Multilingual and Multicultural Development, 9, 3 (1988), pp. 235-247.

VOGEL, T., "Englisch und Deutsch gibt es immer Krieg. Sprachverarbeitungsprozesse beim Erwerb des Deutschen als Drittsprache", Zielsprache Deutsch, 2 (1992), pp. 95-99.

VRIENT, S. de, "Interferenzen einer ersten Fremdsprache beim Erlernen einer zweiten". En: G. Nickel (ed.), Papers from the International Symposium on Applied Contrastive Linguistics, Bielefeld, Cornelson, 1972, pp. 43-50.

WELGE, P. K., "Deutsch nach Englisch. Deutsch als dritte Sprache”. En: S. Ehlers / G. L. Karcher (eds.), Regionale Aspekte des Grundstudiums Germanistik, München, Iudicium, 1987, pp. 189-208. 
WOTJAK, G., "Divergencias y congruencias en el léxico entre el español y el alemán. Los llamados falsos amigos del traductor", Sendebar, 7 (1996), pp. 125-133. 
? 